micina $69 \%$, trimethoprim/sulfametossazolo $99 \%$, teicoplanina $100 \%$.

I risultati relativi al confronto tra la sensibilità ad alcuni antibiotici rilevata nel 1992 e nel 2004 sono riportati di seguito:

\begin{tabular}{lcclcc}
\hline \multicolumn{3}{c}{ Ps.aeruginosa } & \multicolumn{3}{c}{ St.aureus } \\
\hline & 1992 & 2004 & & 1992 & 2004 \\
\hline tobramicina & $85 \%$ & $88 \%$ & penicillina & $5 \%$ & $9 \%$ \\
\hline imipenem & $88 \%$ & $66 \%$ & $\begin{array}{l}\text { meticillina/ } \\
\text { oxacillina }\end{array}$ & $95 \%$ & $67 \%$ \\
\hline ceftazidime & $91 \%$ & $82 \%$ & $\begin{array}{l}\text { tobramicina } \\
\text { clindamicina }\end{array}$ & $95 \%$ & $67 \%$ \\
\hline
\end{tabular}

Conclusioni: la sensibilità di Ps.aeruginosa isolata dai pazienti con fibrosi cistica seguiti presso il nostro centro risulta ancora elevata nei confronti dei principali antibiotici, anche se diminuita rispetto al 1992.

Anche la sensibilità di St.aureus, pur essendo aumentata, rimane su buoni livelli; in particolare l'oxacillino-resistenza resta inferiore al $40 \%$.

\section{SENSIBILITÀ AGLI ANTIBIOTICI DI PS.AERUGINOSA E ST.AUREUS IN PAZIENTI CON FIBROSI CISTICA: CONFRONTO A I 2 ANNI DI DISTANZA}

\author{
Busetti M.*, Macorini D.*, Poli F.**, Serra P.*, Znidarcic C.* \\ *UCO Igiene e Med. Preventiva, Università degli Studi di Trieste; \\ **entro Regionale Fibrosi Cistica, Clinica pediatrica, \\ IRCCS Burlo Garofolo, Trieste
}

Obiettivi: valutare la frequenza delle resistenze ai principali antibiotici dei ceppi di Pseudomonas aeruginosa e Staphylococcus aureus isolati da pazienti con Fibrosi Cistica; confrontare la frequenza dell'antibiotico-sensibilità degli stessi microrganismi a distanza di 12 anni nella popolazione del Centro Fibrosi cistica di Trieste.

Materiali e metodi: sono stati valutati gli antibiogrammi dei ceppi di Ps.aeruginosa e St.aureus isolati da campioni respiratori (escreato o aspirato iopofaringeo) dei pazienti afferenti al Centro Fibrosi cistica dell'IRCCS "Burlo Garofolo" di Trieste nel corso del 2004. Per l'antibiogramma sono stati utilizzati il metodo Kirby-Bauer (per Ps.aeruginosa) e il sistema Vitek2, BioMerieux (per St.aureus). Sono stati presi come confronto i dati relativi agli antibiogrammi degli stessi microrganismi, effettuati mediante sistema di microdiluizione (Sceptor, Becton Dickinson), isolati dai pazienti afferenti al centro FC nell'anno 1992.

Risultati: nel 2004 sono stati isolati 125 ceppi di Ps.aeruginosa e 70 di St.aureus. La frequenza di sensibilità agli antibiotici di Ps.aeruginosa era: ceftazidime $82 \%$, ciprofloxacina $80 \%$, levofloxacina $59 \%$, imipenem $66 \%$, tobramicina $88 \%$, piperacillina $76 \%$, piperacillina/tazobactam $82 \%$. La sensibilità di St.aureus era: penicillina $9 \%$, oxacillina $67 \%$, ciprofloxacina $57 \%$, levofloxacina $73 \%$, tobramicina $67 \%$,clinda- 